\title{
First Spanish series of intestinal transplantation in adult recipients
}

\author{
J. C. Meneu Díaz, E. Moreno González, J. I. García García, A. Moreno, J. C. Montejoํㅡ, F. Colina², \\ B. Pérez, S. Rodríguez ${ }^{3}$, M. Abradelos de Usera, C. Garfia ${ }^{3}$, Y. Fundora, S. Jiménez Galanes, \\ C. Lumbreras ${ }^{4}$, M. León ${ }^{5}$, F. Pérez Cerdá ${ }^{6}$ and J. A. Solís-Herruzo ${ }^{3}$ \\ Services of General Surgery, Digestive Diseases, and Transplantation of Abdominal Organs, ${ }^{1}$ Intensive Medicine, \\ ${ }^{2}$ Pathology, ${ }^{3}$ Digestive Diseases, ${ }^{4}$ Infectious Diseases, ${ }^{5}$ Endocrinology and Nutrition, and ${ }^{6}$ Anesthesiology and \\ Reanimation. Hospital Universitario 12 de Octubre. Madrid, Spain
}

\begin{abstract}
Background: short-bowel transplantation has experienced a substantial growth worldwide following improved results from the late 1990s on, and its coverage by Medicare. According to the International Registry (1985-2005), a total of 1,292 intestinal trasplants for 1,210 patients in 65 hospitals across 20 countries have been carried out thus far.

Objective: to know short-term (6 months) results regarding patient and graft survival from the first Spanish series of intestinal transplants in adult recipients.

Material and methods: we present our experience in the assessment of 20 potential candidates to short-bowel transplantation between June 2004 and October 2005. Of these, 10 patients were rejected and 4 were transplanted, which makes up the sample of our study.

Results: to this date 5 transplants have been carried out in 4 patients ( 2 retransplants, 2 desmoid tumors, 1 short bowel syndrome after excision as a result of mesenteric ischemia). Upon study completion and after a mean follow-up of 180 days (range 90-190 days) all recipients are alive, and all grafts but one $(75 \%)$ are fully operational, with complete digestive autonomy. All patients received induction with alemtuzumab except one, who received thymoglobulin; in all induction was initiated with no steroids.

Conclusions: intestinal transplantation represents a therapeutic option that is applicable in our setting and valid for recipients with an indication who have no other feasible alternative to keep their intestinal failure under control.
\end{abstract}

Key words: Intestinal transplantation. Indications. Selection. Survival.

Recibido: 16-05-06.

Aceptado: 04-09-06.

Correspondencia: Juan Carlos Meneu Díaz. C/ Azalea, 389. Urbanización Sotoazalea. 28109 Alcobendas, Madrid. Fax 9139085 23. e-mail: jcmeneu@hotmail.com
Meneu Díaz JC, Moreno González E, García García JI, Moreno A, Montejo JC, Colina F, Pérez B, Rodríguez S, Abradelos de Usera M, Garfia C, Fundora Y, Jiménez Galanes S, Lumbreras $C$, León M, Pérez Cerdá F, Solís-Herruzo JA. First Spanish series of intestinal transplantation in adult recipients. Rev Esp Enferm Dig 2006; 98: 723-739.

\section{INTRODUCTION}

The first clinical attempts at short-bowel transplantation occurred in Boston in two children back in 1964 (1); in both cases the transplanted organ came fron their mothers. These early transplants were a complete failure, and recipients died in both cases, probably due to improvisation and inadequate preparation.

The fatherhood of intestinal transplantation doubtlessly corresponds to Richard Lillehei. The methodologic rigor in his experimental work, and the accuracy of his clinical observations represent models unequalled thus far. His first transplant, in March 1967, was performed in a 46-year-old woman who had undergone an extended resection of short bowel following extensive mesenteric venous thrombosis. When the procedure was over only the stomach, duodenalpancreatic block, and rectal stump remained. In view of this situation Lillehei decided to intervene and transplant the entire jejunum-ileum from a brain-dead subject. In this first transplant the organ was heterotopically implanted. The graft's inferior mesenteric vein was anastomosed end-side with the left primitive iliac vein, and the superior mesenteric artery laterally with the left primitive iliac artery. The immediate post-operative period was particularly encouraging, as suggested by good graft recoloration and fast peristaltism recovery. Both intestinal ends were brought to the skin in the form of jejunostomy and transverse colostomy. The patient died within a few hours after surgery; the autopsy revealed the presence of extensive portal thrombosis, 
and vena cava thrombosis partially blocking the graft's venous drainage (2).

Since those days when organ transplantation was a heroic act, the introduction of tacrolimus as a basic immunosuppressing agent during the early 1990s led intestinal transplantation to experience a dramatic resurgence primarily under the leadership of the Pittsburgh team, who in 2001 published a pivotal paper discussing their results after 165 transplants in 155 patients. These authors identified 6 factors associated with poorer prognosis, including excessive immunosuppressant doses, steroids, donor and recipient CMV serologic status, and colon segments inclusion, among others (3). The acceptance of recommendations from this work, as well as a number of technical modifications and the introduction of new immunosuppressing agents allowed the inclusion of short-bowel transplant in the clinical setting; intestinal transplantation is nowadays performed in more than 60 hospitals from 20 countries around the world (4).

The International Intestinal Transplant Registry was set up in 1985. From then to this day (1985-2005), according to data presented at the IX International SmallBowel Transplantation Symposium held in Brussels in June 2005, it is estimated that a total of 1,292 intestinal transplants have been carried our worldwide for 1,210 patients. These were isolated small-bowel transplants in most cases, but estimates also include combined intestine and liver, or multivisceral transplants. Results obtained have improved over time particularly in centers with a greater experience, and $75 \%$ of patients have a functioning graft and full digestive autonomy after 6 months.

In our country the first short-bowel transplant in a pediatric recipient was performed at Hospital La Paz on October 21, 1999 by a multidisciplinary team including professionals from Hospital Ramón y Cajal (E. de Vicente y Quijano, et al.) and Hospital La Paz (M. López Santamaría, P. Jara, et al.). Later on, the first Spanish intestine transplant in an adult subject was carried out at Hospital Ramón y Cajal (E. de Vicente y Quijano, et al.) on July 17, 2002.

In our hospital the first intestine transplant took place on December 28, 2004, and 5 further transplants in 4 different patients have been performed since then. We present our team experience, which is so far the greatest cumulative experience with adult recipients in Spain, as ours is presently the only center accredited for this type of transplant on a national basis.

\section{MATERIAL AND METHODS}

Between July 2004 (when the definitive multidisciplinary intestinal transplantation protocol for adult recipients was approved in Hospital 12 de Octubre) and October 2005 we performed a total of 5 transplants (including one second graft, and one third graft) amongst a group of 20 potential candidates assessed.

\section{Characteristics of patients assessed}

This study included all adults assessed in our center as potential candidates to isolated intestinal, hepatointestinal, or multivisceral transplantation. Mean age of patients assessed was $37,07 \pm 8,16$ years $(37 \pm 8,77$ years in the transplanted group; $\mathrm{p}=0,415)$, and $70 \%$ were females (60\% in the transplanted group; $p=0,414)$ (Table I).

All of them were always assessed by the same medical team (surgeons, gastroenterologists, infectologists, and dieticians) (Fig. 1).

Table I. Characteristics of patients evaluated and transplanted

$\begin{array}{lc}\text { Period analyzed } & \text { July } 2004-\text { October } 2005 \\ \text { Patients evaluated } & 20 \\ \text { Mean age (years) } & 37,07 \pm 8,16 \\ \text { Patients transplanted } & 5 \\ \text { Adults /children } & 5 / 0 \\ \text { Males/females } & 14 / 6 \\ \text { No. of prior surgical procedures } & 4 \pm 2 \\ \text { Total ischemia (minutes) } & 360 \text { (range, 300-540) } \\ \text { Warm ischemia (minutes) } & 50 \text { (range,45-65) } \\ \text { Cross testing: performed/positive } & 5 / 2 \\ \text { Anti-CD52 induction therapy } & 5 \\ \text { Donor/Recipient CMV serology } & 5 / 5 \\ \text { D+/R+ } & 5 \\ \text { D+/R- } & 0 \\ \text { D-/R+ } & 0 \\ \text { D-/R- } & 0\end{array}$

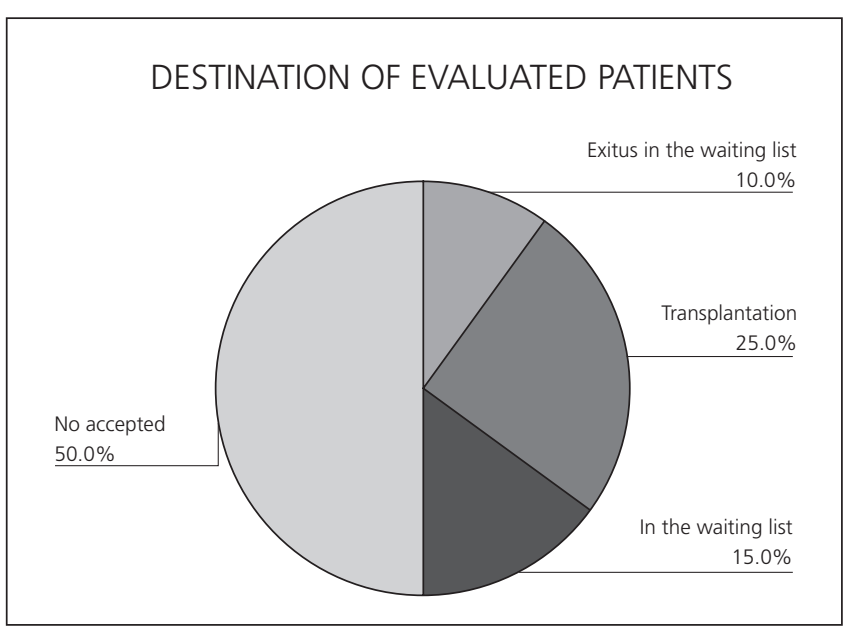

Fig. 1.- Evaluated and transplanted patients in Hospital 12 de Octubre (07/2004-10/2005).

Pacientes evaluados y trasplantados de intestino en el Hospital 12 de Octubre (07/2004-10/2005)

\section{Intestinal transplant indications}

Overall two situations lead to irreversible intestinal failure, and thus warrant intestinal transplantation: a) short-bowel syndrome; and b) intestinal malabsorption syndrome (Table II). 
Table II. Indications for intestinal transplantation

\begin{tabular}{|c|c|}
\hline In children & $\begin{array}{l}\text { Short-bowel syndrome } \\
\text { Volvulus } \\
\text { Gastroschisis } \\
\text { Necrotizing enterocolitis } \\
\text { Intestinal atresia } \\
\text { Intestinal motility disorders } \\
\text { Hirschprung } \\
\text { Chronic idiopathic intestinal } \\
\text { pseudo-obstruction syndrome } \\
\text { Disorders of the intestinal mucosa }\end{array}$ \\
\hline In adults & $\begin{array}{l}\text { Short-bowel syndrome } \\
\text { Mesenteric ischemia } \\
\text { Volvulus } \\
\text { Abdominal trauma } \\
\text { Crohn's disease } \\
\text { Non-resectable (desmoid) tumors } \\
\text { Actinic enteritis }\end{array}$ \\
\hline
\end{tabular}

In our center intestinal transplantation is indicated for (adult) patients with intestinal failure (inability to maintain nutrition and/or water-electrolyte balance) that is chronic and irreversible, who depend on total parenteral nutrition (TPN), and who meet at least one of the following criteria: a) absence of a central venous access (usually from thrombosis) for continued TPN administration; $b$ ) life-threatening relapsing sepsis episodes and/or sepsis associated with TPN administration. We consider sepsis common in the following situations: two or more sepsis episodes per year, or a fungemia, septic shock and/or adult respiratory distress event; c) presence of some underlying condition with life-threatening water-electrolyte loss leading to dehydration in case of TPN discontinuation; and d) presence of serious TPN-associated liver disease (TPNALD) characterized byr cholestasis with or without fibrosis or liver cirrhosis. A diagnosis of TPNALD was established in the following circumstances: hyperbilirubinemia (> $3 \mathrm{mg} / \mathrm{dl}$ ) and/or hypertransaminemia, splenomegaly, thrombopenia, coagulopathy, stoma bleeding, liver fibrosis or cirrhosis.

\section{Surgical technique and donor selection criteria}

All patients in the series received an isolated intestinal transplant (Fig. 2). When necessary the transplant was performed following the excision of a mesenteric desmoid tumor (Fig. 3). The graft was removed from the ideal donor after perfusion with University of Wisconsin solution (Fig. 4). The criteria included in our protocol for short-bowel donor acceptance were as follows: a) age younger than 40 years; b) ICU stay shorter than a week; c) hemodynamic stability (hourly diuresis $>100 \mathrm{ml}$; mean blood pressure $>120 \mathrm{mmHg}$; $\mathrm{O}_{2}$ saturation $>95 \%$; $\mathrm{pO}_{2}>100 \mathrm{mmHg}$ with $\mathrm{FiO}_{2}=1$ and PEEP $<5 \mathrm{mmHg}$; d) absence of prolonged hypotension $(\mathrm{SBP}<80 \mathrm{mmHg}$ );
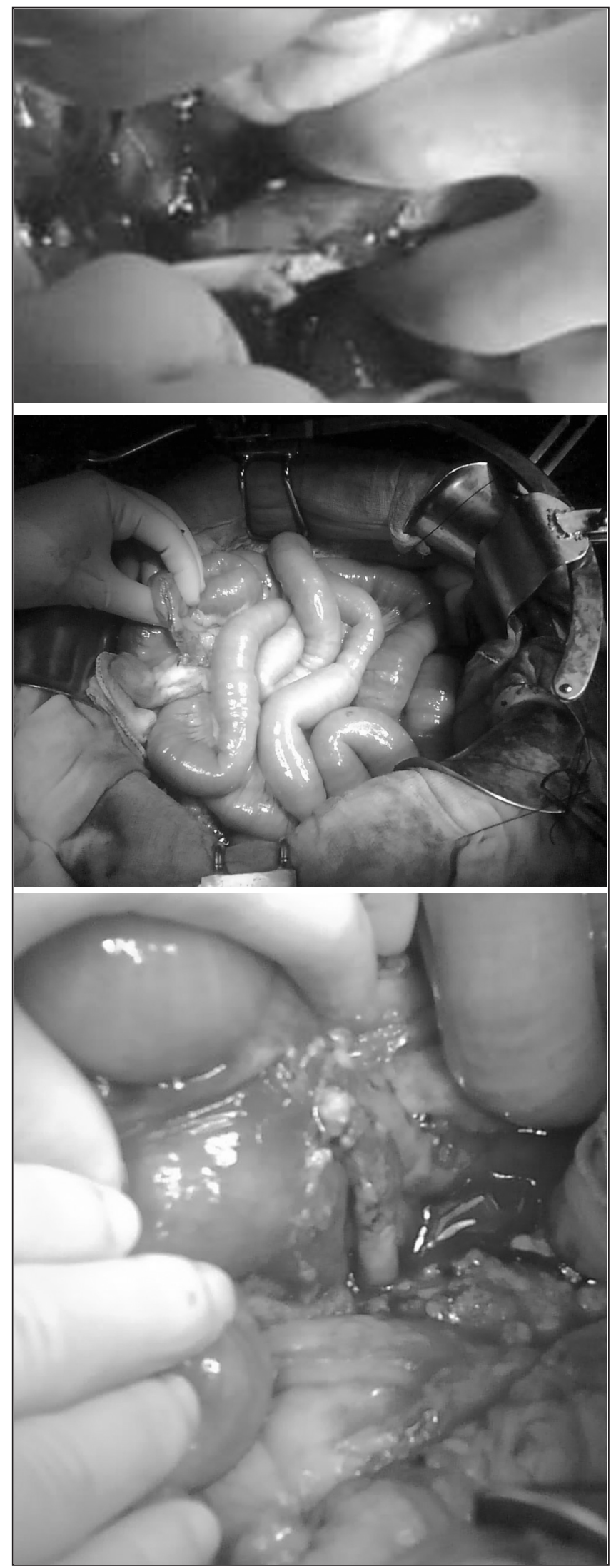

Fig. 2.- Vascular anastomosis in intestinal transplantation. Anastomosis vasculares en el trasplante de intestino. 


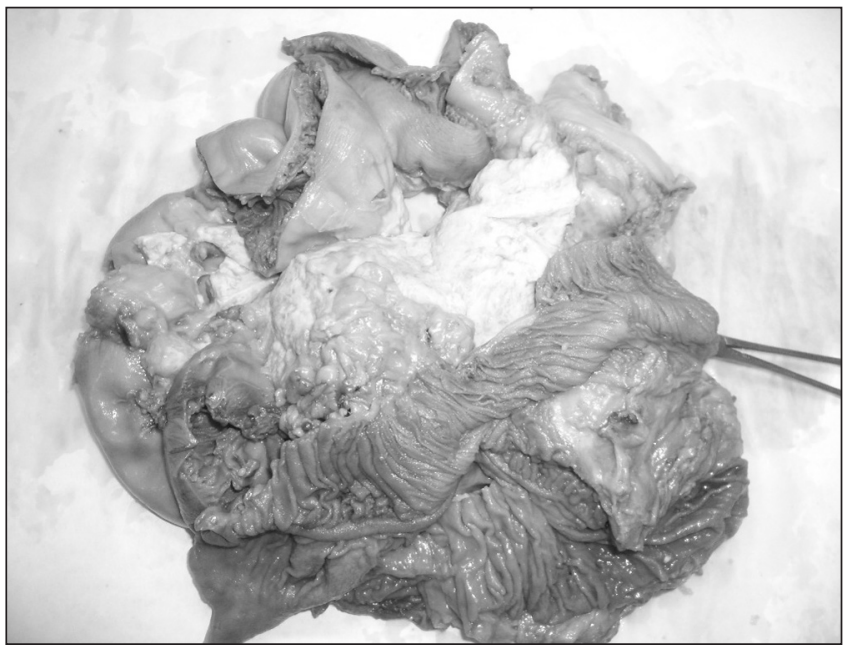

Fig. 3.- Total enterectomy for a desmoid tumor.

Pieza de exéresis tras enterectomía total por tumor desmoide.

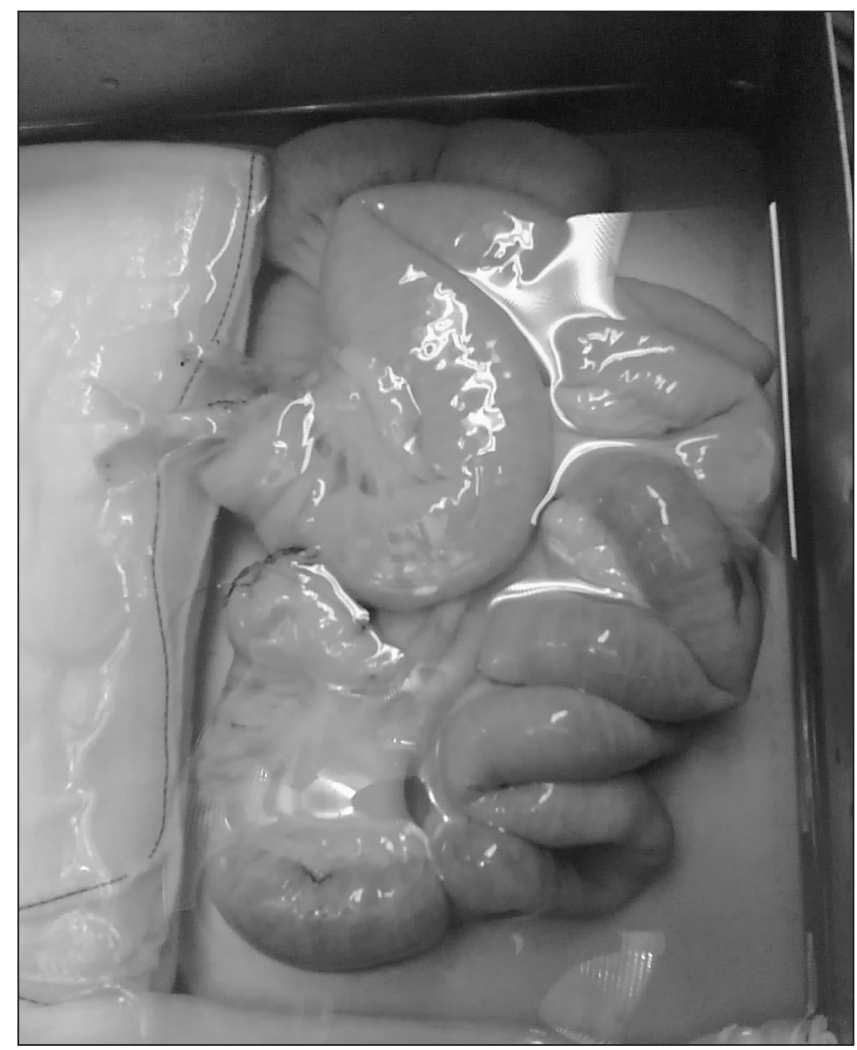

Fig. 4.- Intestinal greft.

Injerto intestinal en banco.

e) absence of cardio-respiratory arrest; f) estimated donor weight between 50 and $75 \%$ of receptor size; g) ABO and $\mathrm{Rh}$ identity; h) ideally absence of CMV; and i) ideally though not mandatory a negative cross-test.

A maximum reduction of graft cold and warm ischemia time is essential. This is why, in an attempt to keep total (cold + warm) ischemia under 8 hours, the recipient is taken to the operating theater to start the procedure and reduce cold ischemia time as soon as the donor surgeon considers the intestinal graft adequate. Once the intestinal graft from an ideal donor arrives in hospital workbench (ex situ, ex vivo) surgery commences (Fig. 4). During this procedure the duodenum is excised, and the superior mesenteric artery (in its aortic origin) and porta vein are isolated and identified, with the latter being sectioned immediately distal to its junction with the splenic vein.

Once the vena cava and the aorta are identified in the retroperitoneum the recipient undergoes arterial and venous anastomoses, and then graft reperfusion. In all cases gastrointetsinal continuity is restored by side-to-side duodenum (recipient) to jejunum (donor) anastomosis, and the graft's distal ileum is exteriorized by performing a terminal ileostomy in the right iliac fossa (Fig. 5). Closure is performed by means of a DUAL mesh to prevent a compartmental syndrome.

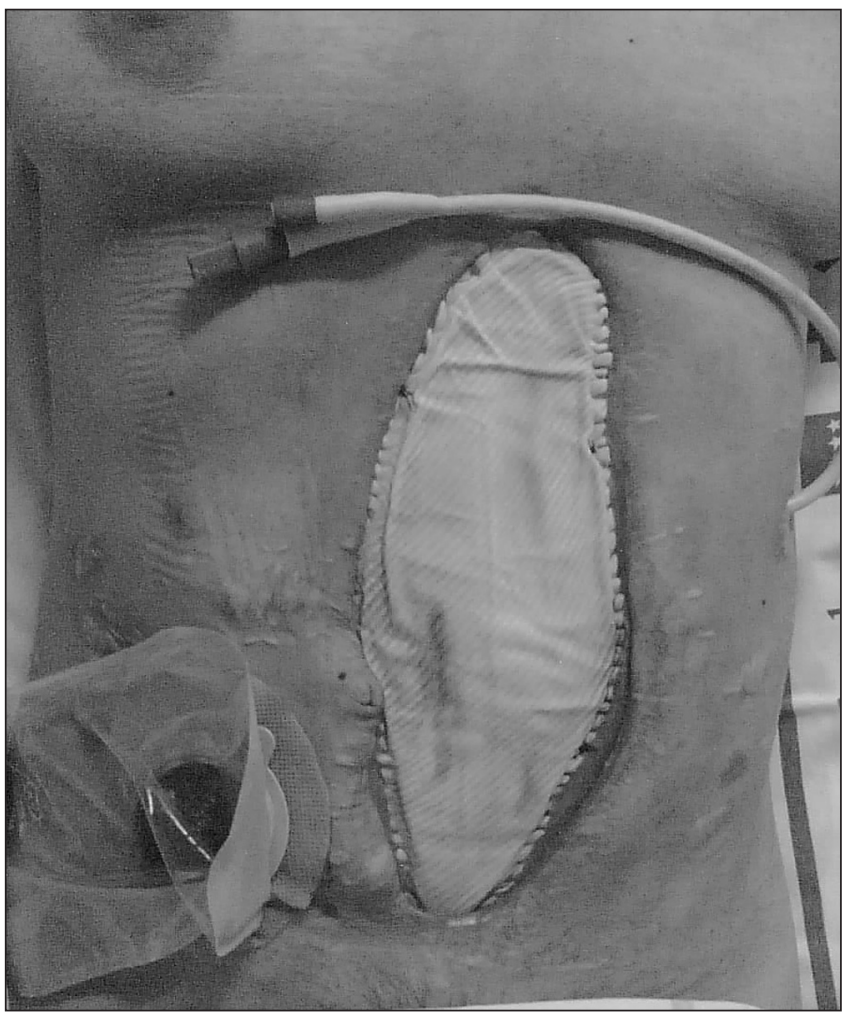

Fig. 5.- Closure of the abdominal wall using a DUAL mesh. lleostomy in right iliac fossa.

Cierre de la pared abdominal mediante malla DUAL. Ileostomía en fosa iliaca derecha.

\section{Immunosuppression protocol}

In our department, of all immunosuppression regimens acceptable for intestinal transplantation it was dual therapy (alemtuzumab or thymoglobulin + tacrolimus) that was adopted in the absence of steroids for the induction or maintenance phase, except in case of acute rejection or 
Table III. Management of acute rejection according to histologic grade. A protocolized biopsy schedule

\begin{tabular}{ll}
\hline \multicolumn{1}{c}{ Acute rejection grade } & \multicolumn{1}{c}{ Treatment } \\
\hline Grade 0: no evidence of rejection & No treatment \\
Grade IND: indeterminate for rejection & No treatment \\
Grade 1: mild rejection & Steroids: \\
& $\bullet 1 \mathrm{~g} /$ day for 3 days, and then tapering down to: \\
& $\bullet 200 \mathrm{mg}, 160 \mathrm{mg}, 120 \mathrm{mg}$, \\
Grade 2: moderate rejection & : $80 \mathrm{mg}, 40 \mathrm{mg}, 20 \mathrm{mg}$ \\
Grade 3: severe rejection & OKT3: $10 \mathrm{mg} / \mathrm{IV} /$ day for 7 days \\
Post-transplant time & Alemtuzumab 30 mg single dose \\
\hline First quarter & Frequency of biopsies per protocol \\
Second quarter & 2 weekly \\
Third quarter & 1 weekly \\
Fourth quarter & 1 fortnightly \\
During second year & 1 monthly \\
During third year & 1 quarterly \\
From fourth year on & 1 half-yearly \\
\hline
\end{tabular}

positive cross testing. Acute rejection was treated according to its grade (Table III).

1. Mabcampath (of choice): mabcampath $1 \mathrm{H}$ is an antiCD52 (a receptor present in all lymphocytes $-\mathrm{T} / \mathrm{B}, \mathrm{NK}-$ and monocytes) antibody that will not affect neutrophils or red blood cells. Its administration entails complete, sustained depletion for at least 6 months. Afterwards a trend towards normalization may be observed, even though baseline levels are never recovered again. Methylprednisolone $1 \mathrm{~g}$ IV, dexchlorpheniramine maleate $5 \mathrm{mg} \mathrm{IV}$, and acetaminophen $1 \mathrm{~g}$ IV are administered before transplantation, followed one hour later by alemtuzumab (Mabcampath $\left.1 \mathrm{H}^{\mathrm{MR}}\right) 30 \mathrm{mg}$ IV over 1 hour (induction).

2. Tacrolimus (FK-506): tacrolimus (Prograf ${ }^{\mathrm{MR}}$ ) is administered in continuous IV infusion at an initial dose of 1 $\mathrm{mg} / 24 \mathrm{~h}$, aiming at sustained optimal levels of $8-12 \mathrm{ng} / \mathrm{dL}$ ( $<6$ months) and 7-10 ng/dL ( $<6$ months). It may be administered via the jejunostomy starting on the first week.

\section{Intestinal graft monitoring}

Intestinal graft monitoring is performed using endoscopic biopsies obtained through the ileostomy orifice, with a minimum of 3 samples from different levels starting at the stoma's distal end. Table III lists the frequency of these biopsies according to our protocol. In addition we perform a histological surveillance of the intestinal mucosa when patient clinical status so advices (suspected rejection: decreased flow through ileostomy, diarrhea, fever, etc.) or in the presence of intestinal rejection. In the latter case we perform such controls at least twice a week.

We categorize acute graft rejection according to guidelines established in the VIII International Small-Bowel Transplantation Symposium, which are based on intestinal biopsy results. Table III lists these criteria, and therapy as chosen for each entry.
Infectious disease prophylaxis: table IV summarizes the protocol followed by us in our center to prevent bacterial, fungal, and viral infection, as well as a vaccination schedule, and a protocol for early infection detection.

\section{Study design and statistical analysis}

This is an observational, prospective study resulting from the practical application of a small-bowel transplantation program based on experimental and clinical experience as gathered both in our country and the USA. Variables (both qualitative and quantitative) regarding pre-, post-, and intra-operative studies were collected for analysis from a prospective database that was set up at baseline for the intestinal and multivisceral transplantation program. Similarly, a careful review of patient medical records was performed in search of relevant data, both in the surgical narrative and daily progress notes, or in reports from endoscopies to rule out acute rejection. The descriptive study of qualitative variables was performed using percentage values, and that of quantitative variables using mean and standard deviation values. Comparisons between groups of qualitative variables were carried out using the chi-squared test, and those for quantitative variables using Student's t-test. No survival probability (Kaplan Meier) studies could be performed given the small number of events (graft or patient loss) in each group.

\section{RESULTS}

\section{Epidemiology and etiology of intestinal failure}

This study includes the first 20 patients who were assessed in our department between July 2004 and October 2005 (Table I, Fig. 1). Mean age was 37,07 \pm 8,16 years, 
Table IV. Prophylaxis of infection in intestinal and multivisceral transplantation

1.- Prevention of bacterial infection

1.1. Perioperative prophylaxis

Piperacillin/Tazobactam $4.5 \mathrm{~g} / \mathrm{IV} / \mathrm{q} 8 \mathrm{~h}$ from anesthetic induction to 5 days after surgery

In case of allergy to betalactams: teicoplanin 400 mg/IV/q12 h + ciprofloxacin 400 mg/IV/q12 h + metronidazole 500 mg/q8 h

1.2. Prophylaxis for intestinal biopsy

Metronidazole $500 \mathrm{mg} / \mathrm{IV}$ + cefepime $1 \mathrm{~g} / \mathrm{IV}$ to be administered within 30 minutes prior to biopsy

1.3. Tuberculosis:

Preoperative Mantoux in all patients. No routine chemoprophylaxis with isoniazide

2.- Prevention of fungal infection

Fluconazole $400 \mathrm{mg} / \mathrm{IV}$ or PO according to tolerance/q24 $\mathrm{h}$ from day +1 to day +14

3.- Prevention of infection with cytomegalovirus and Epstein-Barr virus

D+/R-: ganciclovir IV $5 \mathrm{mg} / \mathrm{kg} / 12 \mathrm{~h}$ adjusted to renal function from day +1 to day +21 . Then valganciclovir $900 \mathrm{mg} /$ day to $\mathrm{month}+6+\mathrm{speci-}$ fic anti-CMV immunoglobulin $100 \mathrm{mg} / \mathrm{kg} / \mathrm{IV}$ every two weeks starting on surgery day and then through to month six

$\mathrm{R}+$ : ganciclovir IV $5 \mathrm{mg} / \mathrm{kg} / 12 \mathrm{~h}$ adjusted to renal function from day +1 to day +21 . Then valganciclovir $900 \mathrm{mg} /$ day/PO through month six D-/R-: blood derivative transfusion using a WBC filter. If in doubt, act as for D+/R-status

For seronegative recipients regarding Epstein-Barr virus, we recommend that demised patients with a positive serology for said virus should not be considered donors

4.- Prevention of $P$. carinii infection

Septrin Forte 1 t/day PO on M, W, F for 12 months starting with patient tolerance to oral ingestion

5.- Vaccination program

Influenza yearly. Pneumococcus (conventional) every 5 years. Hepatitis B virus before transplantation. Complete tetanus vaccination when necessary; varicella vaccination for $\operatorname{lgG}(-)$ recipients

6.- Monitoring infectious complications

6.1. CMV: samples for CMV-related antigenemia measurements will be collected once a week for the initial 3 months, and then every 15 days through to 1 year after transplantation

6.2. EBV: samples will be drawn every fortnight to measure EBV viral load using PCR for the first 6 months, and then monthly through to 1 year after transplantation

6.3. Diarrhea: in case of emergent diarrhea episodes fecal samples will be obtained for Clostridium difficile toxin identification, enteral pathogen culturing, and fecal parasite screening

6.4. Fever: for emergent fever the following will be performed unless otherwise specified: blood culture, urine culture, and blood CMV antigen detection

and $70 \%$ were females. Seventy percent of patients assessed (Fig. 6) had a short-bowel syndrome secondary to massive intestinal resection in the setting of mesenteric thrombosis (30\%), Crohn's disease (5\%), or other causes $(20 \%)$. On the other hand $30 \%$ had a non-resectable desmoid tumor without mesenteric root excision. Following the evaluation period $50 \%$ of patients were considered eligible for intestinal transplantation.

\section{TPN and related complications}

A total of 14 patients $(70 \%)$ required TPN at assessment; two of them had normal laboratory tests and a liver biopsy with nonspecific histologic changes; two had TPN-related cirrhosis; two more had TPN-associated cholestasis, and eight had biochemical cytolysis and cholestasis with no histologic impact.

In those with TPN the mean number of catheter-related sepsis episodes was 1,7 $\pm 1,5 /$ year (range, 0-5/year), which in half of them was serious and prior to assessment for intestinal transplantation. Seven patients had dehydration events despite TPN, with a mean number of serious episodes of 1,30 $\pm 1,34 /$ year (range, $0-4 /$ year). Finally 2 patients were transplanted (one had a histological cholestasis, and one had a normal biopsy).

\section{Post-assessment outcome. Time in the waiting list}

Upon intestinal transplant candidate protocol completion $50 \%$ of patients were considered adequate for the procedure and -following the signing of the required informed consent form- included in the waiting list. Considering the total number of patients who visited our department, $10 \%$ died while in the waiting list for a transplant; $15 \%$ are still waiting, and $25 \%$ have received their transplants. Mean waiting time prior to transplantation was $3,60 \pm 1,14$ months, which is significantly different $(\mathrm{p}=0,005)$ from what happened with the nontransplanted deceased ( $7 \pm 1,41$ months), or with patients still awaiting their chance $(2,33 \pm 0,58$ months $)$. 


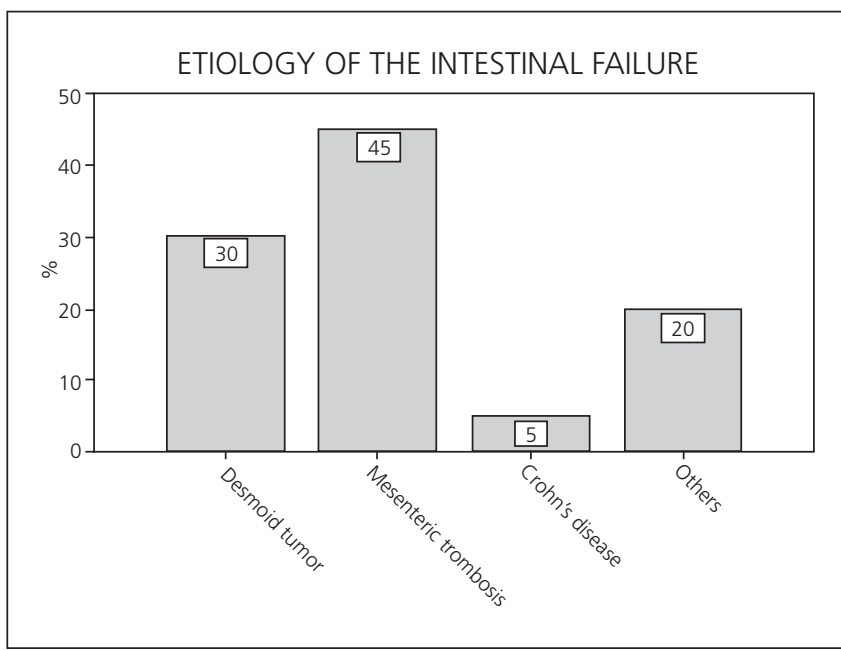

Fig. 6.- Etiology of the intestinal failure in evaluated patients $(07 / 2004-$ 10/2005)

Etiología del fracaso intestinal en los pacientes evaluados (07/200410/2005).

\section{Donor characteristics}

In all cases donors offered by Organización Nacional de Trasplantes (ONT) were assessed according to the aforementioned protocol. Mean donor age was 14 years (range, 12-36 years), median weight was $35 \mathrm{~kg}$ (range, $37-60 \mathrm{~kg}$ ), and median ICU stay was 2 days (range, 0-4 days). All grafts were perfused using Wisconsin solution, and in all cases multiple organs were extracted, with the following organs being obtained and then implanted (at receptor hospitals): liver, heart, both kidneys, intestine, pancreas (in one case) and lungs (in two cases). Median cold ischemia time was 360 minutes (range, 300-540 minutes), and mean warm ischemia time was 50 minutes (range, 45-65 minutes).

\section{Intestinal graft vascularization, reconstruction of digestive continuity, and abdominal wall closure}

In all cases the aorta and subrenal vena cava were dissected to perform vascular anastomoses (Fig. 2). Arterialization was carried out with an end-side mesenteric-aortic anastomosis; the graft's venous drainage was directed to the systemic circulation using an end-side portal-cava anastomosis. The proximal digestive anastomosis (sideto-side duodeno-jejunostomy) was performed using nonresorbable suture with interrupted 4/0-gauge stitches. No distal digestive anastomoses were performed, and a terminal ileostomy was deliberately established in the right iliac fossa, through which per-protocol endoscopies for the screening of acute rejection were carried out. Currently after a median follow-up of 180 days (range, 90-190 days), all patients have a terminal ileostomy, and none of them as yet been closed. On two occasions the abdominal wall was primarily closed, and three patients required biomaterials (meshes) to prevent an intraabdominal compartmental syndrome (Fig. 5).

\section{Postoperative outcome}

All patients are alive ( $0 \%$ mortality), and all grafts but one are functioning (75\% intestinal graft functioning) (Fig. 7). Graft carriers maintain their digestive autonomy, which was reached after a median postoperative period of 33 days (range, 28-35 days). Mean ICU stay was 2 days (range, 2-45 days), and patients were thence transferred to an intermediate care unit associated with the Digestive and General Surgery Department, with a median stay of 20 days there (range, 16-23 days). Median in-hospital stay was 160 days (range, 75-190 days). On study completion, of all 5 transplanted patients 3 are still in hospital and 2 were discharged after an overall stay of 75 and 170 days, respectively.

In 3 cases (60\%) rejection episodes were diagnosed. These were grade-II rejections in two patients (effectively treated with OKT3 $10 \mathrm{ml} / \mathrm{IV} / 24$ hours for 10 days), and a grade-III event in one patient, which was refractory to OKT3 and alemtuzumab, and led to intestinal graft excision.

Regarding post-transplant infectious complications, the presence of 1,02 $\pm 1,03$ episodes of central catheter infection was established during the first postoperative month. On day 45 post-transplant one patient had a serious CMV infection reactivation (which remitted following therapy with IV ganciclovir). Another patient had an intraabdominal Candida infection in the sixth postoperative week, which required surgical drainage by laparotomy.

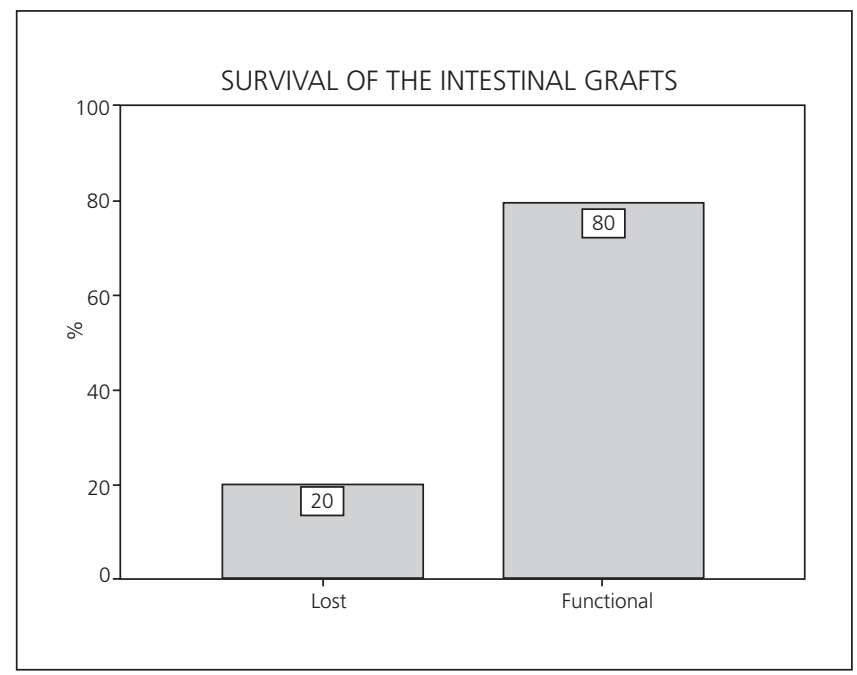

Fig. 7.- Survival of grafts in intestinal transplanted patients in Hospital 12 de Octubre (07/2004-10/2005).

Superviviencia del injerto en trasplantados de intestino en el Hospital 12 de Octubre (07/2004-10/2005). 


\section{DISCUSSION}

Intestinal failure, defined as the inability to maintain an adequate nutritional status in the absence of TPN, occurs in the adult because of two major reasons (5): a) intestinal malabsorption syndrome (actinic enteritis or chronic pseudoobstruction); and b) short-bowel syndrome (SBS).

SBS is the predominating cause, and is secondary to massive intestinal resections due to mesenteric ischemia, trauma, Crohn's disease or intestinal or mesenteric tumors (desmoid tumors). Therefore, SBS occurs when a patient (either congenitally or from surgical procedures) keeps only $200 \mathrm{~cm}$ of functioning small bowel (6).

Nutrient absorption is closely related to total absorption surface, so the risk for intestinal failure development also depends on the presence or absence of additional large bowel. It is estimated that for a variable period of 1 to 2 years the remaining intestine may adjust to the new status using villous hypertrophy, which would increase both its diameter and length thus leading to increaased nutrient absorption. An oral diet promotes this process, and a longer intestine as well as the presence of a healthy small bowel and colon are facilitating factors. Thus it is commonly established that patients with a higher risk include: a) those with duodenostomy or jejuno-ileal anastomosis with only $35 \mathrm{~cm}$ of bowel remaining; b) those with jejuno-colic or ileo-colic anastomosis and a remnant shorter than $60 \mathrm{~cm}$; and c) those with a terminal jejunostomy with less than $115 \mathrm{~cm}$ of functioning intestine.

The most relevant aspect in the medical management of patients with short-bowel syndrome is the adequate provision of all micronutrients, macronutrients, and fluids necessary to prevent energy malnutrition, specific nutrient deficiencies, and dehydration, in addition to correcting water-electrolyte and acid-base disorders (7). Thus, the development of TPN during the early 1960s opened up an era of new therapeutic possibilities for patients with chronic intestinal failure. Priot to this most patients died from malnutrition and dehydration; the development of food formulations for intravenous administration, together with the development of central, long-term vascular accesses, was the cornerstone for TPN implementation, first within hospitals and then at home. In fact survivals with TPN for up to 30 years have been reported. However, TPN entails a number of complications that render its long-term use an impossible goal in a considerable amount of patients.

TPNALD (8) occurs from $3 \%$ of patients at 5 years to $15 \%$ at 19 years; however, some authors have reported a prevalence as high as $50 \%$ at 6 years. Although related mortality is significant $(12,5 \%)$, what is truly relevant is that $50 \%$ of patients dying while on TPN pass away because of TPNALD. In addition, $100 \%$ of patients with TPNALD die within 10,8 \pm 7 months of plasma bilirubin increase.
Catheter-related venous thrombosis (9) represents 12\% of those associated with TPN (66\% are asymptomatic and identified on catheter change). The incidence of thrombosis is 0,2 episodes/1,000 catheters/day, and the management of repeat episodes makes oral anticoagulation mandatory, which is particularly difficult to manage due to inadequate dicoumarin absorption and bleding risk.

Central catheter-associated sepsis (10) may threaten the patient's life. Its prevalence has been established in 0,8 infections/1,000 catheters/year in adults, and represents $61 \%$ of TPN-associated complications. It accounts for $50 \%$ of TPN-related deaths; furthermore, repeat infection contribute to TPNALD progression.

Given this situation the role of surgery for patients with SBS may be included within three categories: a) intestinal continuity restoration using ileo/jejuno-colic anasomoses to facilitate adjustment, and to potentially cancel TPN dependence; b) procedures directed to duplicate the absorptive surface based on the remaining small bowel segment (Bianchi's technique); and c) intestinal (multivisceral) transplantation.

To this day intestinal transplantation has been carried out for patients developing complications attributable to intestinal failure or TPN-derived complications. In the USA Medicare (11) approved small-bowel transplantation coverage for patients failing TPN as of October 2000, which has definitely contributed to the establishment of this therapeutic modality.

The first intestinal transplant for an adult patient in our hospital took place in December 2004. Ours is thus a young program, but one that accumulates the largest experience in adults on a national basis. In scarcely one year we have assessed some twenty patients referred from other sites as potential intestinal transplant recipients. These were mostly women younger than 40 years, which is consistent with reports from other international centers with a longer experience.

We rejected $50 \%$ of transplant candidates, and $50 \%$ of those accepted have been transplanted thus far. These data reflect the dramatic potential for expansion that this procedure enjoys in our country, which has the highest donation rates worldwide $(34,6 \mathrm{dmp}$ in 2004). Most transplanted patients $(70 \%)$ had SBS, but in contrast with other teams we assessed a high percentage (30\%) of patients harboring a desmoid tumor. In such cases the transplant was performed immediately after tumor resection, thus avoiding anenteric waiting time, TPN-derived problems, and forfeited right of domicile, which would otherwise occur in two-phase treatments, i.e., tumor excision followed a few months later by the small-bowel transplant.

Similarly we saw that a high percentage of patients assessed (70\%) were TPN-dependent; it is among these that we found TPN-associated complications such as infection, venous thrombosis, and TPNALD. Furthermore, serious dehydration episodes occurred in $50 \%$ of patients despite TPN, with a mean of 1,3 episodes, which clearly 
reflects the difficulties of water-electrolyte balance provision to avoid intestinal transplantation.

Given that our team is the only one accredited in Spain for adult recipients, and considering the high donation rate in our country, patients were transplanted after waiting for hardly 4 months. This is in contrast with reports by other groups, where waiting time lasted for up to 12 months. This fact offers a number of benefits: a) it allowed us to keep the indication for isolated intestinal transplant; b) combined liver-intestine transplants for TPN-associated cirrhosis have not been necessary; and c) it allowed us to set aside organs, particularly the liver, for other patients in the waiting list. For this reason we deem it relevant that specialists involved in the management of patients with short-bowel syndrome (surgeons, gastroenterologists, dieticians, endocrinologists, etc.) should consider the potential indication of intestinal transplantation earlier in the course of disease, to optimize patient status in terms of post-transplant outcome.

According to data reported at the I FESNAD Congress (12), a total of 86 patients visiting 17 hospitals were on home TPN in our country. Therefore, the number of patients receiving a transplant in one year hardly accounts for one third of those who could have benefited from the procedure.

From a technical standpoint all of our patients had a portocava shunt performed, as the native liver received splanchnic blood flow via the splenic and gastric tributary veins. Theoretically a portal drainage would be more physiological, and would ensure the hepatotrophic effects of direct intestinal blood flow through the liver. However, numerous papers published during the last decade, both in humans and experimental animals, question the functional, metabolic, and immune superiority of this vein anastomosis modality $(13,14)$. In fact, Berney et al. showed no significant differences between caval and portal drainage in terms of mortality, morbidity, rejection, functionality, and both patient and graft survival (15).

The most widely accepted way of transit restoration following a transplant includes a proximal (duodeno-jejunal) and a distal ("funnel ileostomy") anastomosis (Bishoop-Koop) (15). In our series this reconstruction was in all cases performed using a side-to-side duodenojejunal anastomosis, and a terminal ileostomy in the right iliac fossa to facilitate endoscopic and biopsic monitoring. No distal anastomosis was made during the first surgery, and it was postponed until ileostomy closure. In this way problems arising from potential dehiscence in the immediate post-transplant period are prevented.

Abdominal wall closure following transplantation is still concerning because of the so-called loss of the right of domicile by intestinal contents, and the risk for an intraabdominal compartmental syndrome $(16,17)$. This is the reason why in patients where a primary wall closure could not be carried out a prosthetic material was used, which was then sequentially reduced until closure completion. No relevant complications occurred with relation to the use of said materials.
Rejection has been traditionally considered the most serious concern in small-bowel transplantation $(18,19)$. It still is nowadays, but rejection rates and severity have been dramatically decreased with the use of tacrolimus and most especially monoclonal antibodies (alemtuzumab, thymoglobulin). According to our experience $60 \%$ of cases had a rejection event (3 patients); 2 were put under control with OKT3; the third patient was refractory to this compound and alemtuzumab, and thus required graft excision.

At the end of the present study, and after a median follow-up of 180 days (range, 90-190 days), all grafts but one remain functional $(75 \%)$, and no patient has died. These figures are consistent with data reported in the literature, and with those recorded at the International Intestinal Transplant Registry. All patients have digestive autonomy, and their postoperative stay was prolonged due to problems deriving from abdominal wall closure.

Therefore, while aware of our series' small size, we believe this is merely the beginning of a new therapeutic option in our country; it shows that intestinal transplantation is here to stay, and should be offered to selected patients meeting a specific number of criteria. These include patients depending on TPN but who from TPN developed serious complications precluding TPN persistence; patients who, as a result, have very limited possibilities for survival.

\section{ACKNOWLEGEMENTS}

This study was supported in part by grants 012/2004 and 08/2005 from "Fundation Mutua Madrileña", Madrid, Spain.

\section{REFERENCES}

1. Mittal NK, Tzakis AG, Kato T, Thompson JF, et al. Current status of small bowel transplantation in children: update 2003. Pediatr Clin North Am 2003; 50: 1419-33.

2. Todo S, Tzakis AG, Abu-Elmagd K, Reyes J, Nakamura K, Casavilla A, et al. Intestinal transplantation in composite visceral grafts or alone. Ann Surg 1992; 216: 223-33

3. Abu-Elmagd K, Reyes J, Bond G, Mazariegos G, Wu T, Murase N, et al. Clinical intestinal transplantation: a decade of experience at a single center. Ann Surg 2001; 234: 404-16.

4. Small Bowell Transplant Registry. IXth Internacional Small Bowell Transplantation Symposium, Brussels, June 30-July 2005.

5. Abu-Elmagd K, Reyes J, Todo S, Rao A, Lee R, Irih W, et al. Clinical intestinal transplantation: new perspectives and immunologic considerations. J Am Coll Surg 1998; 186: 512-25.

6. American Gastroenterological Association Medical Position Statement: Short Bowel Syndrome and Intestinal Transplantation. Gastroenterology 2003; 124: 1105-10.

7. Van Gossum A, Vahedi K, Abdel-Malik, Staun M, Pertkiewicz M, Shaffer J, et al. Clinical, social and rehabilitation status of long-term home parenteral nutrition patients: results of a European multicentre survey. Clin Nutr 2001; 20: 205-10.

8. Chan S, McCowen KC, Bistrian BR, Thibault A, Keane-Ellison M, Forse RA, et al. Incidence, prognosis and etiology of end-stage liver disease in patients receiving total parenteral nutrition. Surgery 1999 ; 126: $28-34$

9. DeCicco M, Matovic M, Balestreri L, Panarello G, Fantin D, Morassut $\mathrm{S}$, et al. Central venous thrombosis: an early and frequent compli- 
cation in cancer patients bearing long-term silastic catheters. A prospective study. Thromb Res 1998; 86: 101-13.

10. Planas M, Castella M, León M y Grupo NADYA: Nutrición Parenteral Domiciliaria (NPD): registro NADYA; 2000.

11. Disponible en: http://www.cms.hhs.gov/manuals/pm_trans/AB00130. pdf. These are the Medicare guidelines for coverage of intestinal transplantation

12. Registro de la Nutrición Parenteral Domiciliaria (NPD) del año 2003. Planas Vila M, Grupo NADYA. Nutrición Hospitalaria. Vol. XX • Suplemento 1; 2005.

13. Liu H, Teraoka S, Kawai T, Hayashi T, Fuchinove S, Nozawa M, et al. Comparative evaluation of systemic vs. portal venous drainage in long-term surviving rats with orthotopic small bowel transplantation. Transplant Proc 1992; 24: 1505-6.

14. Stratta R, Shokouh-Amiri MH, Egidi MF, Grewal HP, Kizilisik AT, Nezakatgco N, et al. A prospective comparison of simultaneous kidney-pancreas transplantation with systemic-enteric versus portal-en- teric venous drainage. Ann Surg 2001; 233: 740-51.

15. Bueno J, Abu-Elmagd K, Mazariegos G, Madariaga J, Fung J, Reyes $\mathrm{J}$, et al. Composite liver-small bowel allografts with preservation of donor duodenum and hepatic biliary system in children. J Pediatr Surg 2000; 35: 291-6.

16. Alexandrides IJ, Liu P, Marshall DM, Nery JR, Tzakis AG, Thaller $\mathrm{SR}$, et al. Abdominal wall closure after intestinal transplantation. Plast Reconstr Surg 2000; 106: 805.

17. Levi DM, Tzakis AG, Kato T, Madariaga J, Mittal NK, Nery J, et al. Transplantation of the abdominal wall. Lancet 2003; 361: 2173 6.

18. Tzakis AG, Nery JR, Thompson J, Webb MG, Khan FA, Khan RT, et al. New immunosuppressive regimens in clinical intestinal transplantation. Transplant Proc 1997; 29: 683-5.

19. Abu-Elmagd K, Fung J, McGhee W, Martin D, Mazariegos G, Schaefer $\mathrm{N}$, et al. The efficacy of daclizumab for intestinal transplantation: preliminary report. Transplant Proc 2000; 32: 1195-6. 